\title{
FRICTIONAL HEATING WITH TIME-DEPENDENT SPECIFIC POWER OF FRICTION
}

\author{
Katarzyna TOPCZEWSKA*
}

${ }^{*}$ Faculty of Mechanical Engineering, Department of Mechanics and Applied Computer Science, Bialystok University of Technology, ul. Wiejska 45C, Bialystok 15-351, Poland

k.topczewska@doktoranci.pb.edu.pl

received 25 October 2016, revised 11 May 2017, accepted 15 May 2017

\begin{abstract}
In this paper analytical solutions of the thermal problems of friction were received. The appropriate boundary-value problems of heat conduction were formulated and solved for a homogeneous semi-space (a brake disc) heated on its free surface by frictional heat fluxes with different and time-dependent intensities. Solutions were obtained in dimensionless form using Duhamel's theorem. Based on received solutions, evolution and spatial distribution of the dimensionless temperature were analyzed using numerical methods. The numerical results allowed to determine influence of the time distribution of friction power on the spatio-temporal temperature distribution in brake disc.
\end{abstract}

Key words: Brake Disc, Frictional Heating, Temperature, Time-Dependent Power of Friction

\section{INTRODUCTION}

Frictional characteristics and wear in an disc-pad contact area are changing significantly depending on the way of absorbed heat energy by a braking system. In some cases the major part of braking work is accomplished in the initial stage of braking, in other cases work is more evenly distributed in time. Essential influence on value and evolution of the temperature has the nature of change with time the specific power of friction. Classification of the time courses of specific power of friction during single braking was proposed in monograph (Chichinadze, 1967). Table 5.3 on page 78 in this study contains eleven different functions, which describe change of the specific power of friction with braking time. Analysis of the influence of the each time course on the temperature were conducted based on, received by author solution to the one-dimensional thermal problem of friction for semispace. However, these solutions were found with simplifying assumptions, which refer to interior points of the half-space on the axis perpendicular to the outer surface. It was assumed that, the temperature is proportional to the braking time and the temperature increments and also the average volumetric temperature are equal. The studies reviews of accurate (without mentioned above simplifications) solutions to the one-dimensional thermal problems of friction are in monographs (Jewtuszenko et al., 2014, Kuciej, 2012). They demonstrate that the vast majority of the received accurate solutions usually concern only two elementary cases from the above mentioned table. In first of them, specific power of friction in the braking process has constant value, in second it decreases linearly from maximum value in the initial moment to zero in the standstill. The second time course of specific power of friction is realizable during braking with constant deceleration and an immediate increase of pressure to nominal value in the initial time moment. Influence of the time of the contact pressure increase to nominal value on the temperature distribution was investigated in the article (Topczewska, 2016).
In this study the following simplification was adopted: a brake disc was replaced by homogeneous semi-space and the friction process of contacting bodies was replaced by heating process on outer surface by heat flux with given intensity (Evtushenko et al., 2007, Matysiak et al., 2007, Yevtushenko et al., 2005-2007, 2009-2012, 2014). Assuming one-dimensional direction of heat conduction is correct for high values of the Peclet number (for high velocity of braking). Taking into account mentioned above assumptions, analytical solutions to the one-dimensional thermal problems of friction for three time courses of specific power of friction (VI, VIII and XI positions in table 5.3 in monograph (Chichinadze, 1967)) were determined analytically. They reflect braking modes until the vehicle standstill.

\section{STATEMENT OF THE PROBLEM}

A considered semi-space orientation is given relative to a Cartesian frame of reference Oxyz with origin placed on the free surface (z-axis is vertical to this plane). The half-space $\mathrm{z} \geq 0$ is heated on its outer surface $z=0$ by heat flux with timedependent $0<\mathrm{t}<\mathrm{t}_{\mathrm{s}}$ intensity $\mathrm{q}_{\mathrm{i}}(\mathrm{t})=\mathrm{q}_{0} \mathrm{q}_{\mathrm{i}}^{*}(\mathrm{t}), \mathrm{i}=1,2,3$ (Fig. 1). Intensity of heat fluxes is proportional to the specific power of friction (Ling, 1973). Therefore, omitting constant proportional factor, the so-called heat partition ratio, the following three dimensionless time courses of friction power (Chichinadze, 1967):

$$
\begin{aligned}
& q_{1}^{*}(t)=3\left(1-\frac{t}{t_{s}}\right)^{2}, q_{2}^{*}(t)=6 \frac{t}{t_{s}}\left(1-\frac{t}{t_{s}}\right), \\
& q_{3}^{*}(t)=6\left(\sqrt{\frac{t}{t_{s}}}-\frac{t}{t_{s}}\right), \quad 0 \leq t \leq t_{s} .
\end{aligned}
$$

were considered.

Functions (1) are selected such that, the following braking work density: 
$w_{i}(t)=\int_{0}^{t} q_{i}(s) d s, 0 \leq t \leq t_{s}, i=1,2,3$,

in moment of standstill is constant and equal to $\mathrm{w}_{\mathrm{i}}\left(\mathrm{t}_{\mathrm{s}}\right)=\mathrm{q}_{0} \mathrm{t}_{\mathrm{s}}$. Graphs of dimensionless braking work density evolutions $\mathrm{w}_{\mathrm{i}}^{*}(\mathrm{t})=\mathrm{w}_{\mathrm{i}}(\mathrm{t}) /\left(\mathrm{q}_{0} \mathrm{t}_{\mathrm{s}}\right)$ were presented in Fig. 2.

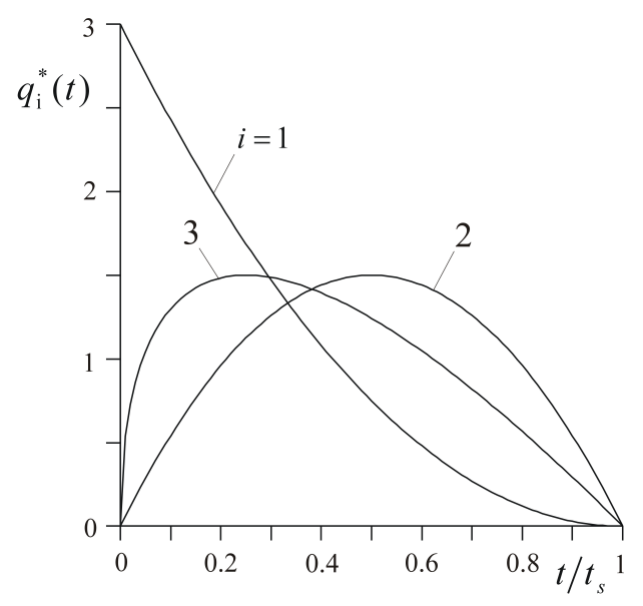

Fig. 1. The time courses of the dimensionless specific power of friction $q_{i}^{*}(t), i=1,2,3(1)$

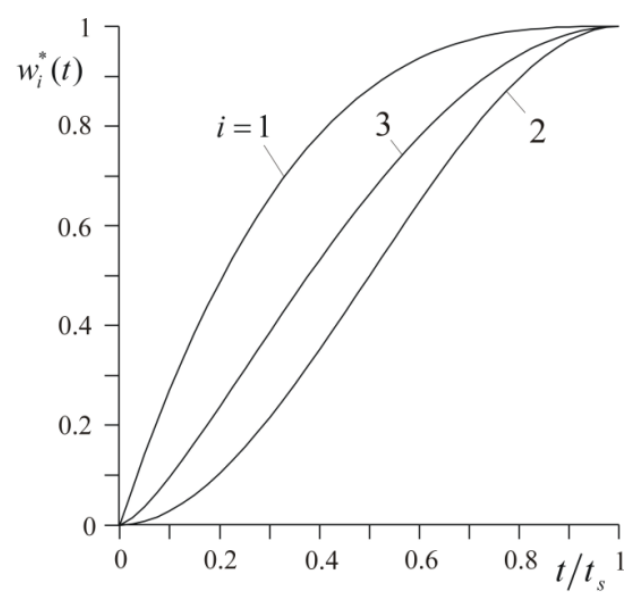

Fig. 2. The time courses of the total dimensionless braking work density $w_{i}^{*}(t), i=1,2,3(2)$

Distributions of the one-dimensional transient dimensionless temperature fields $T_{i}^{*}(\zeta, t), i=1,2,3$ in semi-space were found from solution to the following boundary-value problem of heat conduction, formulated in dimensionless form:

$\frac{\partial^{2} T_{i}^{*}(\zeta, \tau)}{\partial \zeta^{2}}=\frac{\partial T_{i}^{*}(\zeta, \tau)}{\partial \tau}, \zeta \geq 0,0 \leq \tau \leq \tau_{S}$

$\left.\frac{\partial T_{i}^{*}(\zeta, \tau)}{\partial \zeta}\right|_{\zeta=0}=-q_{i}^{*}(\tau), i=1,2,3,0 \leq \tau \leq \tau_{s}$,

$T_{i}^{*}(\zeta, \tau) \rightarrow 0, \zeta \rightarrow \infty, 0 \leq \tau \leq \tau_{s}$,

$T_{i}^{*}(\zeta, 0)=0, \zeta \geq 0$,

where

$\zeta=\frac{z}{a}, \tau=\frac{k t}{a^{2}}, \tau_{s}=\frac{k t_{s}}{a^{2}}, T_{0}=\frac{q_{0} a}{K}, T_{i}^{*}=\frac{T_{i}-T_{a}}{T_{0}}$,

$\mathrm{a}=\sqrt{3 \mathrm{kt}_{\mathrm{s}}}$ is effective depth of the heat penetration inside brake disc (Chichinadze, et al., 1979), $\mathrm{T}_{\mathrm{a}}$ is initial temperature, $\mathrm{K}, \mathrm{k}$ are thermal conductivity and thermal diffusivity, respectively.

\section{SOLUTION OF THE PROBLEM}

Solutions to the boundary-value problem (3)-(6) were found based on Duhamel's theorem (Ozisik,1993):

$T_{i}^{*}(\zeta, \tau)=\int_{0}^{t} q_{i}^{*}(s) \frac{\partial}{\partial \tau} T^{*(0)}(\zeta, \tau-s) d s, \zeta \geq 0,0 \leq \tau \leq \tau_{s}$,

where (Carslaw H. S. et al. 1959):

$T^{*(0)}(\zeta, \tau)=2 \sqrt{\tau} \operatorname{ierfc}\left(\frac{\zeta}{2 \sqrt{\tau}}\right), \zeta \geq 0,0 \leq \tau \leq \tau_{s}$,

is solution to the problem (3)-(6) with constant in time $q^{*}(\tau)=1$ intensity of heat flux in boundary condition (4).

Taking into account value of the following derivative of the complementary error function (Abramowitz M. et al. 1972):

$\frac{\partial}{\partial x} \operatorname{erfc}(x)=-\frac{2 x}{\sqrt{\pi}} e^{-x^{2}}$,

the following partial derivative was counted:

$\frac{\partial}{\partial \tau}\left[2 \sqrt{\tau-s} \operatorname{ierfc}\left(\frac{\zeta}{2 \sqrt{\tau-s}}\right)\right]=\frac{e^{-\left(\frac{\zeta}{2 \sqrt{\tau-s}}\right)^{2}}}{\sqrt{\pi(\tau-s)}}$.

Substituting relation (9) and derivative (11) to the formula (8), we received:

$$
\begin{aligned}
& T_{1}^{*}(\zeta, \tau)=3 \int_{0}^{\tau}\left(1-\frac{s}{\tau_{s}}\right)^{2} \frac{e^{-\left(\frac{\zeta}{2 \sqrt{\tau-s}}\right)^{2}}}{\sqrt{\pi(\tau-s)}} d s, \zeta \geq 0,0 \leq \tau \leq \tau_{s}, \\
& T_{2}^{*}(\zeta, \tau)=6 \int_{0}^{\tau}\left[\frac{s}{\tau_{s}}-\left(\frac{s}{\tau_{s}}\right)^{2}\right] \frac{e^{-\left(\frac{\zeta}{2 \sqrt{\tau-s}}\right)^{2}}}{\sqrt{\pi(\tau-s)}} d s, \zeta \geq 0,0 \leq \tau \leq \tau_{s}, \\
& T_{3}^{*}(\zeta, \tau)=6 \int_{0}^{\tau}\left(\sqrt{\frac{s}{\tau_{s}}}-\frac{s}{\tau_{s}}\right) \frac{e^{-\left(\frac{\zeta}{2 \sqrt{\tau-s}}\right)^{2}}}{\sqrt{\pi(\tau-s)}} d s, \zeta \geq 0,0 \leq \tau \leq \tau_{s} .
\end{aligned}
$$

Above equations (12)-(14) were written as difference integrals:

$T_{1}^{*}(\zeta, \tau)=3\left[I_{0}(\zeta, \tau)-I_{2}(\zeta, \tau)\right], \zeta \geq 0,0 \leq \tau \leq \tau_{s}$,

$T_{2}^{*}(\zeta, \tau)=6\left[I_{1}(\zeta, \tau)-I_{2}(\zeta, \tau)\right], \zeta \geq 0,0 \leq \tau \leq \tau_{s}$,

$T_{3}^{*}(\zeta, \tau)=6\left[I_{1 / 2}(\zeta, \tau)-I_{1}(\zeta, \tau)\right], \zeta \geq 0,0 \leq \tau \leq \tau_{s}$,

where

$$
\begin{aligned}
& I_{0}(\zeta, \tau)=\frac{1}{\sqrt{\pi}} \int_{0}^{\tau} \frac{1}{\sqrt{\tau-s}} e^{-\left(\frac{\zeta}{2 \sqrt{\tau-s}}\right)^{2}} d s, \\
& I_{1}(\zeta, \tau)=\frac{2}{\tau_{s} \sqrt{\pi}} \int_{0}^{\tau} \frac{s}{\sqrt{\tau-s}} e^{-\left(\frac{\zeta}{2 \sqrt{\tau-s}}\right)^{2}} d s, \\
& I_{2}(\zeta, \tau)=\frac{1}{\tau_{s}^{2} \sqrt{\pi}} \int_{0}^{\tau} \frac{s^{2}}{\sqrt{\tau-s}} e^{-\left(\frac{\zeta}{2 \sqrt{\tau-s}}\right)^{2}} d s, \\
& I_{1 / 2}(\zeta, \tau)=\frac{1}{\sqrt{\tau_{s} \pi}} \int_{0}^{\tau} \sqrt{\frac{s}{\tau-s}} e^{-\left(\frac{\zeta}{2 \sqrt{\tau}-s}\right)^{2}} d s .
\end{aligned}
$$

Using substitution $x=1 / \sqrt{\tau-s}$, the integrals (18)-(21) were designated:

$$
\begin{aligned}
& I_{0}(\zeta, \tau)=\frac{2}{\sqrt{\pi}} L_{2}(\zeta, \tau), \\
& I_{1}(\zeta, \tau)=\frac{2}{\tau_{s} \sqrt{\pi}}\left[\tau L_{2}(\zeta, \tau)-L_{4}(\zeta, \tau)\right], \\
& I_{2}(\zeta, \tau)=\frac{2}{\tau_{s}^{2} \sqrt{\pi}}\left[\tau^{2} L_{2}(\zeta, \tau)-2 \tau L_{4}(\zeta, \tau)+L_{6}(\zeta, \tau)\right],
\end{aligned}
$$

where 
$L_{k}(\zeta, \tau)=\int_{\frac{1}{\sqrt{\tau}}}^{\infty} e^{-\left(\frac{\zeta}{2}\right)^{2} x^{2}} \frac{d x}{x^{k}}, k=2,4,6$

Based on recurrence relation (Prudnikov et al., 1986):

$\int \frac{e^{-(a x)^{2}}}{x^{n}} d x=-\frac{e^{-(a x)^{2}}}{(n-1) x^{n-1}}-\frac{2 a^{2}}{n-1} \int \frac{e^{-(a x)^{2}}}{x^{n-2}} d x, a>0$,

$n=2,3, \ldots$,

and the following solution (Prudnikov et al., 1986):

$L_{2}(\zeta, \tau)=\sqrt{\pi \tau} \operatorname{ierfc}\left(\frac{\zeta}{2 \sqrt{\tau}}\right)$

we achieved:

$L_{4}(\zeta, \tau)=\frac{\tau \sqrt{\pi \tau}}{3}\left\{\left[1-2\left(\frac{\zeta}{2 \sqrt{\tau}}\right)^{2}\right] \operatorname{ierfc}\left(\frac{\zeta}{2 \sqrt{\tau}}\right)+\frac{\zeta}{2 \sqrt{\tau}} \operatorname{erfc}\left(\frac{\zeta}{2 \sqrt{\tau}}\right)\right\}$,

$L_{6}(\zeta, \tau)=\frac{\tau^{2} \sqrt{\pi \tau}}{5}\left\langle\left\{1-\frac{2}{3}\left(\frac{\zeta}{2 \sqrt{\tau}}\right)^{2}\left[1-2\left(\frac{\zeta}{2 \sqrt{\tau}}\right)^{2}\right]\right\} \operatorname{ierfc}\left(\frac{\zeta}{2 \sqrt{\tau}}\right)+\right.$

$\left.+\frac{\zeta}{2 \sqrt{\tau}} \operatorname{erfc}\left(\frac{\zeta}{2 \sqrt{\tau}}\right)\left[1-\frac{2}{3}\left(\frac{\zeta}{2 \sqrt{\tau}}\right)^{2}\right]\right\rangle$

Taking into account results (27)-(29) in equations (22)-(24), we obtain:

$I_{0}(\zeta, \tau)=2 \sqrt{\tau} \operatorname{ierfc}\left(\frac{\zeta}{2 \sqrt{\tau}}\right)$,

$I_{1}(\zeta, \tau)=\frac{2 \tau \sqrt{\tau}}{3 \tau_{s}}\left\{2\left[1+\left(\frac{\zeta}{2 \sqrt{\tau}}\right)^{2}\right] \operatorname{ierfc}\left(\frac{\zeta}{2 \sqrt{\tau}}\right)+\frac{\zeta}{2 \sqrt{\tau}} \operatorname{erfc}\left(\frac{\zeta}{2 \sqrt{\tau}}\right)\right\},(31)$

$I_{2}(\zeta, \tau)=\frac{2 \sqrt{\tau}}{15}\left(\frac{\tau}{\tau_{s}}\right)^{2}\left\{\left[8+18\left(\frac{\zeta}{2 \sqrt{\tau}}\right)^{2}+4\left(\frac{\zeta}{2 \sqrt{\tau}}\right)^{4}\right] \operatorname{ierfc}\left(\frac{\zeta}{2 \sqrt{\tau}}\right)-\right.$

$\left.-\frac{\zeta}{2 \sqrt{\tau}} \operatorname{erfc}\left(\frac{\zeta}{2 \sqrt{\tau}}\right)\right\}$

Subsequently integral (21) will be counted. Using the same substitution $x=1 / \sqrt{\tau-S}$, we have:

$I_{1 / 2}(\zeta, \tau)=\sqrt{\frac{\tau}{\tau_{s} \pi}} J(\zeta, \tau)$

where

$J(\zeta, \tau)=\int_{\frac{1}{\sqrt{\tau}}}^{\infty} \sqrt{x-\frac{1}{\tau}} e^{-\left(\frac{\zeta}{2}\right)^{2} x} \frac{d x}{x^{2}}$.

Integrating (34) by parts, we have:

$J(\zeta, \tau)=\frac{1}{2} J_{1}(\zeta, \tau)-\frac{\zeta^{2}}{4} J_{2}(\zeta, \tau)$,

where

$J_{1}(\zeta, \tau)=\int_{\frac{1}{\sqrt{\tau}}}^{\infty} \frac{1}{\sqrt{x-\tau^{-1}}} e^{-\left(\frac{\zeta}{2}\right)^{2} x} \frac{d x}{x}$,

$J_{2}(\zeta, \tau)=\int_{\frac{1}{\sqrt{\tau}}}^{\infty} \sqrt{x-\tau^{-1}} e^{-\left(\frac{\zeta}{2}\right)^{2} x} \frac{d x}{x}$.

Based on the following formula (Prudnikov et al., 1998):

$\int_{u}^{\infty} \frac{(x-u)^{v} e^{-\mu x}}{x} d x=u^{v} \Gamma(v+1) \Gamma(-v, u \mu)$,

$u>0, \operatorname{Re} v>-1, \operatorname{Re} \mu>0$,

functions (36) and (37) were written in the forms:

$J_{1}(\zeta, \tau)=\sqrt{\tau} \Gamma\left(\frac{1}{2}\right) \Gamma\left(\frac{1}{2}, \frac{\zeta^{2}}{4 \tau}\right)$,

$J_{2}(\zeta, \tau)=\frac{1}{\sqrt{\tau}} \Gamma\left(\frac{3}{2}\right) \Gamma\left(-\frac{1}{2}, \frac{\zeta^{2}}{4 \tau}\right)$.

Taking into account relation (Prudnikov et al., 1998):

$\Gamma(v+1, z)=v \Gamma(v, z)+z^{v} e^{-z}$, we receive:

$\Gamma\left(-\frac{1}{2}, \frac{\zeta^{2}}{4 \tau}\right)=2 \sqrt{\pi}\left(\frac{\zeta}{2 \sqrt{\tau}}\right)^{-1} \operatorname{ierfc}\left(\frac{\zeta}{2 \sqrt{\tau}}\right)$.

In regard to the following (Prudnikov et al., 1998):

$\Gamma\left(\frac{1}{2}\right)=\sqrt{\pi}, \Gamma\left(\frac{3}{2}\right)=\frac{\sqrt{\pi}}{2}, \Gamma\left(\frac{1}{2}, \frac{\zeta^{2}}{4 \tau}\right)=\sqrt{\pi} \operatorname{erfc}\left(\frac{\zeta}{2 \sqrt{\tau}}\right)$,

and (42), we can write:

$J_{1}(\zeta, \tau)=\pi \sqrt{\tau} \operatorname{erfc}\left(\frac{\zeta}{2 \sqrt{\tau}}\right)$,

$J_{2}(\zeta, \tau)=\frac{\pi}{\sqrt{\tau}}\left(\frac{\zeta}{2 \sqrt{\tau}}\right)^{-1} \operatorname{ierfc}\left(\frac{\zeta}{2 \sqrt{\tau}}\right)$.

Using solutions (44) and (45), we write (35) in form:

$J(\zeta, \tau)=\frac{\pi \sqrt{\tau}}{2}\left[\operatorname{erfc}\left(\frac{\zeta}{2 \sqrt{\tau}}\right)-2\left(\frac{\zeta}{2 \sqrt{\tau}}\right) \operatorname{ierfc}\left(\frac{\zeta}{2 \sqrt{\tau}}\right)\right]$,

Substituting above formula to equation (33), we receive:

$I_{1 / 2}(\zeta, \tau)=\frac{\tau}{2} \sqrt{\frac{\pi}{\tau_{s}}}\left[\operatorname{erfc}\left(\frac{\zeta}{2 \sqrt{\tau}}\right)-\frac{\zeta}{\sqrt{\tau}} \operatorname{ierfc}\left(\frac{\zeta}{2 \sqrt{\tau}}\right)\right]$.

Having regard relations (30)-(32) and (47) we determine searched temperature fields from (15)-(17):

$T_{1}^{*}(\zeta, \tau)=2 \sqrt{\tau}\left\langle\frac{1}{5}\left(\frac{\tau}{\tau_{s}}\right)^{2}\left[8+18\left(\frac{\zeta}{2 \sqrt{\tau}}\right)^{2}+4\left(\frac{\zeta}{2 \sqrt{\tau}}\right)^{4}\right] \operatorname{ierfc}\left(\frac{\zeta}{2 \sqrt{\tau}}\right)-\right.$ $-\left[7+2\left(\frac{\zeta}{2 \sqrt{\tau}}\right)^{2}\right] \frac{\zeta}{2 \sqrt{\tau}} \operatorname{erfc}\left(\frac{\zeta}{2 \sqrt{\tau}}\right)+3 \operatorname{ierfc}\left(\frac{\zeta}{2 \sqrt{\tau}}\right)-\frac{2 \tau}{\tau_{s}}\{2[1+$

$\left.\left.\left.+\left(\frac{\zeta}{2 \sqrt{\tau}}\right)^{2}\right] \operatorname{ierfc}\left(\frac{\zeta}{2 \sqrt{\tau}}\right)+\frac{\zeta}{2 \sqrt{\tau}} \operatorname{erfc}\left(\frac{\zeta}{2 \sqrt{\tau}}\right)\right\}\right\rangle \zeta \geq 0,0 \leq \tau \leq \tau_{s}$,

$T_{2}^{*}(\zeta, \tau)=4 \sqrt{\tau}\left\langle\frac{\tau}{\tau_{s}}\left\{2\left[1+\left(\frac{\zeta}{2 \sqrt{\tau}}\right)^{2}\right] \operatorname{ierfc}\left(\frac{\zeta}{2 \sqrt{\tau}}\right)-\frac{\zeta}{2 \sqrt{\tau}} \operatorname{erfc}\left(\frac{\zeta}{2 \sqrt{\tau}}\right)\right\}-\right.$ $-\frac{1}{5}\left(\frac{\tau}{\tau_{s}}\right)^{2}\left[8+18\left(\frac{\zeta}{2 \sqrt{\tau}}\right)^{2}+4\left(\frac{\zeta}{2 \sqrt{\tau}}\right)^{4}\right] \operatorname{ierfc}\left(\frac{\zeta}{2 \sqrt{\tau}}\right)-[7+$

$\left.\left.2\left(\frac{\zeta}{2 \sqrt{\tau}}\right)^{2}\right] \frac{\zeta}{2 \sqrt{\tau}} \operatorname{erfc}\left(\frac{\zeta}{2 \sqrt{\tau}}\right)\right\rangle, \zeta \geq 0,0 \leq \tau \leq \tau_{S}$,

$T_{3}^{*}(\zeta, \tau)=3 \tau \sqrt{\frac{\pi}{\tau_{s}}}\left[\operatorname{erfc}\left(\frac{\zeta}{2 \sqrt{\tau}}\right)-\frac{\zeta}{\sqrt{\tau}} \operatorname{ierfc}\left(\frac{\zeta}{2 \sqrt{\tau}}\right)\right]-\frac{4 \tau \sqrt{\tau}}{\tau_{s}}\{2[1+$

$\left.\left.\left(\frac{\zeta}{2 \sqrt{\tau}}\right)^{2}\right] \operatorname{ierfc}\left(\frac{\zeta}{2 \sqrt{\tau}}\right)-\frac{\zeta}{2 \sqrt{\tau}} \operatorname{erfc}\left(\frac{\zeta}{2 \sqrt{\tau}}\right)\right\}, \zeta \geq 0,0 \leq \tau \leq \tau_{s}$.

\section{NUMERICAL ANALYSIS}

Numerical analysis of the temperature distributions in a disc (semi-space) during single braking, for three different time courses of heat flux intensities, was conducted based on the found analytical solutions (48)-(50). The dimensionless input parameters used in calculations are: spatial coordinate $\zeta$, time $\tau$ and braking time $\tau_{\mathrm{s}}=1$, which are defined by formulas (7). According to equations (1) and (2) total amount of thermal energy absorbed by the disc during braking for considered intensities of heat flux is equal.

Evolutions of the dimensionless temperature on the friction surface and selected depths $\zeta$ inside semi-space for three changes with time of heat flux densities (Fig. 2), were shown in Fig. 3. The temperature distribution for intensity of heat flux $q_{1}^{*}(t)$, when its value is the largest on the initial stage of braking and decreases to zero with time, were presented in Fig. 3a. At the beginning of the braking temperature $\mathrm{T}_{1}^{*}$ on outer surface of semi-space $(\zeta=0)$ rapidly increases, to achieve maximum value $\mathrm{T}_{1 \max }^{*}=$ 1.20 in time $\tau / \tau_{s}=0.32$, and then decreases until the end of the process. 

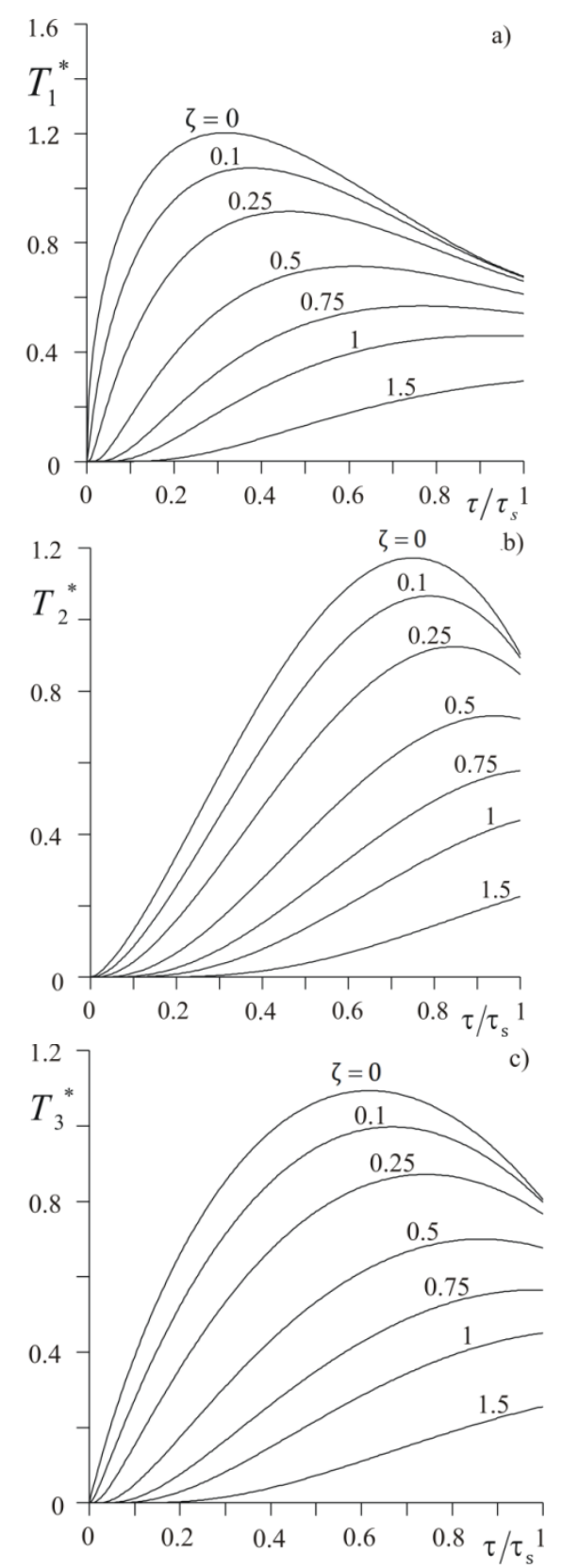

Fig. 3. Evolutions of the dimensionless temperatures a) $T_{1}^{*}$, b) $T_{2}^{*}$, c) $T_{3}^{*}$ on few distances $\zeta$ from the friction surface

In the cases, when in initial stage of braking the heat flux intensity increase reaching maximum value in $25 \%\left(\tau / \tau_{s}=0.25\right.$ for $\left.\mathrm{q}_{3}^{*}(\mathrm{t})\right)$ or $50 \%\left(\tau / \tau_{\mathrm{s}}=0.5\right.$ for $\left.\mathrm{q}_{2}^{*}(\mathrm{t})\right)$, of total braking time, reaching largest temperature value on the working surface also gets longer, and equals to, $\tau / \tau_{\mathrm{s}}=0.75$ for $\mathrm{T}_{2 \max }^{*}=1.17$ (Fig. 3b) and $\tau / \tau_{s}=0.6$ for $\mathrm{T}_{3 \max }^{*}=1.09$ (Fig. 3c), respectively. In both cases after reaching maximum values, the temperature of the surface decreases to the standstill. The dimensionless temperature on the friction surface $\zeta=0$ in the stop time moment $\tau / \tau_{s}=1$ is higher in the case heating disc by heat flux intensity $\mathrm{q}_{2}^{*}(\mathrm{t})$ (Fig. $3 \mathrm{~b}$ ) and $\mathrm{q}_{3}^{*}(\mathrm{t})$ (Fig. $3 \mathrm{c}$ ) compared with temperature value in brake disc heated by flux with intensity $q_{1}^{*}(t)$ (Fig. 3a). In the all considered cases, increasing distance $\zeta$ from the frictional surface (Fig. 3) the temperature value is getting lower and time to reach maximum increases. The monotonically temperature increase during the entire heating process with intensity $\mathrm{q}_{2}^{*}(\mathrm{t})$ can be observed (Fig. $3 b$ ) on the distance $\zeta \geq 0.75$, and with $\mathrm{q}_{1}^{*}(\mathrm{t})$ and $\mathrm{q}_{3}^{*}(\mathrm{t})$ (Fig. $3 \mathrm{a}, 3 \mathrm{c}$ ) under the effective depth of the heat penetration $\zeta \geq 1$.
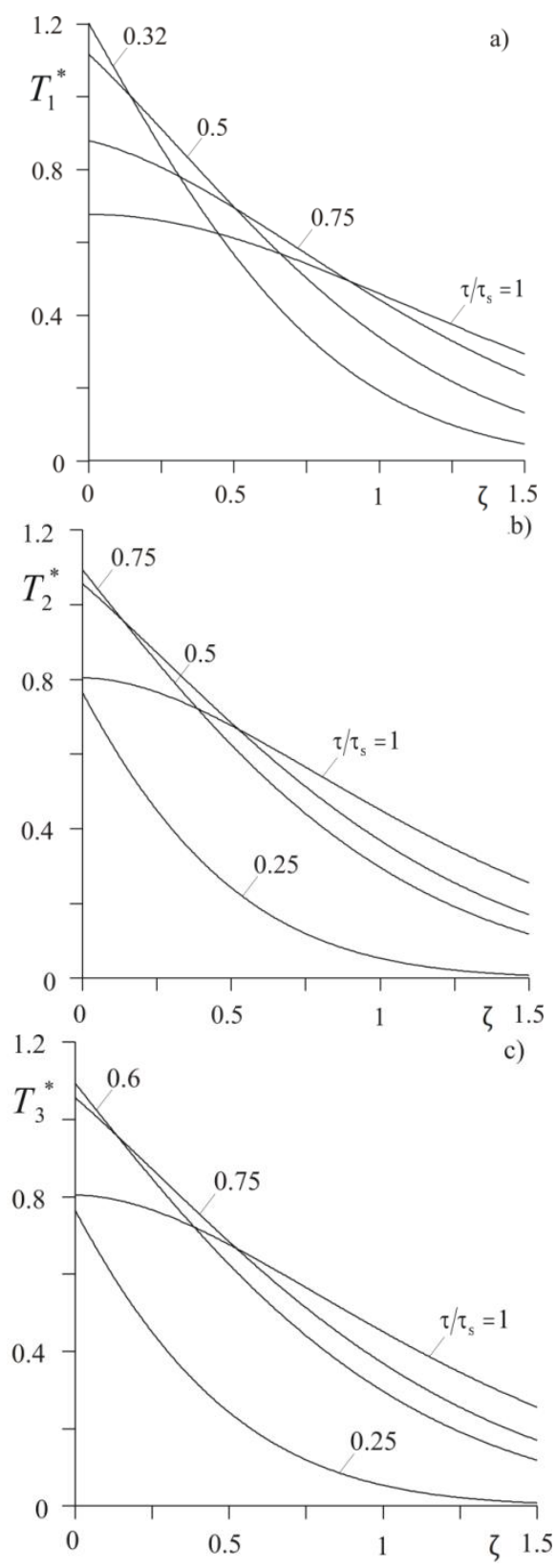

Fig. 4. Distribution of the dimensionless temperatures a) $T_{1}^{*}$, b) $T_{2}^{*}$, c) $T_{3}^{*}$ inside disc at few different dimensionless time moments $\tau$

The dimensionless temperature changes with depth $\zeta$ for different intensities of heat flux $q_{i}^{*}(t), i=1,2,3$ in selected time moments $\tau$, are shown in Fig. 4 . In the all cases, the temperature monotonically decreases with increasing the distance $\zeta$ from heated surface. In the considered distance range $0 \leq \zeta \leq 1.5$, the largest gradients of the temperature inside the disc $\Delta \mathrm{T}_{1}^{*}=$ 1.15 (Fig. 4a), $\Delta \mathrm{T}_{2}^{*}=1.05$ (Fig. 4b) and $\Delta \mathrm{T}_{3}^{*}=0.98$ (Fig. 4c), occurs in the moments of reaching maximum temperature values on the friction surface $\zeta=0$. The most uniform distributions of the temperature in disc are in standstill moment $\tau / \tau_{s}=1$, temperatures on the working surface have lower values $\mathrm{T}_{1}^{*}=0.68$ (Fig. 4a), $\mathrm{T}_{2}^{*}=0.9$ (Fig. 4b) and $\mathrm{T}_{3}^{*}=0.8$ (Fig. 4c). Mentioned gradients reach minimum values $\Delta \mathrm{T}_{1}^{*}=0.38$ (Fig. $4 \mathrm{a}$ ), $\Delta \mathrm{T}_{2}^{*}=0.64$ (Fig. 4b) and $\Delta \mathrm{T}_{3}^{*}=0.54$ (Fig. 4c) in the stop moment. 


\section{CONCLUSIONS}

Analytical solutions to the heat conduction problems for semispace heated on outer surface by heat flux with different time courses of intensity were determined. Numerical analysis of evolution and spatial distribution of the dimensionless temperature (along the distance from the friction surface) were carried out based on obtained solutions. In results the following conclusions were formulated:

- there is a strict relation between the time moment of reaching maximum values of dimensionless temperature on the heated surface and the time moment of maximum intensity occurrence of corresponding heat flux. In the considered cases, extreme temperature value is reached ca. $30 \%$ of braking time later than maximum of the heat flux intensity time function;

- maximum values of dimensional temperature on the friction surface of the disc, in all cases are similar. The largest difference between them is only $9 \%$ of this value. While, in these cases, maximum values of the friction power differ twofold;

- gradients of the dimensionless temperature inside the disc are the lowest in the standstill $\tau / \tau_{s}=1$. The most intense cooling of the outer surface after reached maximum temperature, occurs in the first case $q_{1}^{*}(t)$, while the temperature achieve the largest maximum values.

Nomenclature: a - effective depth of heat penetration [m]; erf(x) Gauss error function; $\operatorname{erfc}(\mathrm{x})=1-\operatorname{erf}(\mathrm{x})-$ complementary error function; $\quad \operatorname{ierfc}(\mathrm{x})=\pi^{-1 / 2} \exp \left(-\mathrm{x}^{2}\right)-\operatorname{xerfc}(\mathrm{x}) \quad-\quad$ integral of complementary error function; $\mathrm{K}-$ thermal conductivity [W K-1 m-1]; $\mathrm{k}$ - thermal diffusivity [m2 s-1]; $\mathrm{q}$ - intensity of the heat flux [W $\mathrm{m}-2$ ] $\mathrm{T}$ - temperature $[\mathrm{K}] ; \mathrm{T}^{*}$ - dimensionless temperature; $\mathrm{T}_{\mathrm{a}}$ - initial temperature $[\mathrm{K}]$; $\mathrm{T}_{0}$-temperature scaling factor $[\mathrm{K}] ; \mathrm{t}-$ time $[\mathrm{s}]$; $\mathrm{t}_{\mathrm{s}}-$ braking time [s]; $\mathrm{w}-$ braking work density [W $\mathrm{m}-2 \mathrm{~s}-1]$; $\mathrm{w}^{*}$ - dimensionless braking work density; xyz - spatial coordinates $[\mathrm{m}] ; \Gamma(\mathrm{v}, \mathrm{z})$ - gamma function; $\tau$ - dimensionless time (Fourier number); $\tau_{s}-$ dimensionless braking time; $\zeta$-dimensionless depth.

\section{REFERENCES}

1. Abramowitz M., Stegun I.A. (1972), Handbook of Mathematical Functions with Formulas, Graphs, and Tables, National Bureau of Standards, Washington.

2. Carlslaw H.S., Jaeger J. C. (1959), Conduction of Heat in Solids, 2nd ed.Clarendon Press, Oxford.

3. Chichinadze A.V. (1967), Estimation and investigation of external friction during braking, Nauka, Moscow. - 231 p. (in Russian).

4. Chichinadze A.V., Braun E.D., Ginsburg A.G., Ignat'eva Z.V. (1979), Calculation, Test and Selection of Frictional Couples, Nauka, Moscow (in Russian).

5. Evtushenko A., Kutsei, M. (2007), Non-stationary frictional heat problem for plane-parallel layer-half-space system, Journal of Friction and Wear, 28(3), 246-259.
6. Jewtuszenko O. (red) (2014), Analytical and numerical modelling of process of transient heat generation in friction components of brake systems, Publisher of Technical University of Bialystok, Bialystok (in Polish).

7. Kuciej M. (2012), Analytical models of transient frictional heating Publisher of Technical University of Bialystok, Bialystok (in Polish).

8. Ling F.F. (1973), Surface Mechanics, Wiley, New York.

9. Matysiak S., Evtushenko O., Kuciej M. (2007),Temperature field in the process of braking of a massive body with composite coating, Materials Science, 43(1), 62-69.

10. Ozisik M.N. (1993), Heat conduction, 2nd Ed. Wiley: New York.

11. Prudnikov A. P., Brychkov Yu. A., Marichev O. I. (1986), Integrals and Series. Vol. 1: Elementary Functions, Gordon and Breach: New York.

12. Prudnikov A.P., Brychkov Yu.A., Marichev O. I. (1998), Integrals and Series. Vol. 2: Special Functions, New York-London: Taylor \& Francis.

13. Topczewska K. (2016), Temperature distribution in a brake disc with variable contact pressure, Technical Issues, 1, 90-95.

14. Yevtushenko A. A., Kuciej M., Różniakowska M. (2005), Thermal cleavage stresses in a piecewise homogeneous plate, Materials Science, 41 (5) 581-588.

15. Yevtushenko A.A., Kuciej M. (2006), Initiating of thermal cracking of materials by frictional heating, J. Friction and Wear, 27(2) 9-16.

16. Yevtushenko A.A., Kuciej M. (2009), Influence of convective cooling on the temperature in a frictionally heated strip and foundation, International Communications in Heat and Mass Transfer, 36(2), 129-136.

17. Yevtushenko A.A., Kuciej M. (2010), Two heat conduction problems with frictional heating during braking, J. Theor. Appl. Mech., 48(2), 367-380.

18. Yevtushenko A.A., Kuciej M., Yevtushenko 0.0. (2011), Temperature and thermal stresses in material of pad during braking, Arch. Appl. Mech., 81(6), 715-726.

19. Yevtushenko A.A., Kuciej M. (2012), One-dimensional thermal problem of friction during braking: The history of development and actual state, International Journal of Heat and Mass Transfer, 55(15), 4148-4153.

20. Yevtushenko A.A., Kuciej M., Yevtushenko O. (2014), The asymptotic solutions of heat problem of friction for a three-element tribosystem with generalized boundary conditions on the surface of sliding, International Journal of Heat and Mass Transfer, 70, 128-136.

21. Yevtushenko A.A., Rozniakowska M., Kuciej M. (2007a), Transient temperature processes in composite strip and homogeneous foundation, International Communications in Heat and Mass Transfer, 34(9), 1108-1118.

22. Yevtushenko A.A., Rozniakowska M., Kuciej M. (2007b), Laserinduced thermal splitting in homogeneous body with coating. Numerical Heat Transfer, Part A: Applications, 52(4), 357-375.

This work is part of the project no. MB/WM/18/2017 which is carried out in the Faculty of Mechanical Engineering, Bialystok University of Technology. 